\title{
Spectral asymmetry of the massless Dirac operator on a 3-torus
}

Article

Accepted Version

Downes, R. J., Levitin, M. and Vassiliev, D. (2013) Spectral asymmetry of the massless Dirac operator on a 3-torus. Journal of Mathematical Physics, 54 (11). 111503. ISSN 00222488 doi: https://doi.org/10.1063/1.4828858 Available at https://centaur.reading.ac.uk/34885/

It is advisable to refer to the publisher's version if you intend to cite from the work. See Guidance on citing.

To link to this article DOI: http://dx.doi.org/10.1063/1.4828858

Publisher: American Institute of Physics

All outputs in CentAUR are protected by Intellectual Property Rights law, including copyright law. Copyright and IPR is retained by the creators or other copyright holders. Terms and conditions for use of this material are defined in the End User Agreement.

\section{www.reading.ac.uk/centaur}

\section{CentAUR}

Central Archive at the University of Reading

Reading's research outputs online 


\title{
Spectral asymmetry of the massless Dirac operator on a 3-torus
}

\author{
Robert J. Downes, ${ }^{1, a)}$ Michael Levitin, ${ }^{2, b)}$ and Dmitri Vassiliev ${ }^{1,(c)}$ \\ 1) Department of Mathematics, University College London, Gower Street, \\ London WC1E 6BT, UK \\ ${ }^{2)}$ Department of Mathematics and Statistics, University of Reading, Whiteknights, \\ PO Box 220, Reading RG6 6AX, UK
}

Consider the massless Dirac operator on a 3-torus equipped with Euclidean metric and standard spin structure. It is known that the eigenvalues can be calculated explicitly: the spectrum is symmetric about zero and zero itself is a double eigenvalue. The aim of the paper is to develop a perturbation theory for the eigenvalue with smallest modulus with respect to perturbations of the metric. Here the application of perturbation techniques is hindered by the fact that eigenvalues of the massless Dirac operator have even multiplicity, which is a consequence of this operator commuting with the antilinear operator of charge conjugation (a peculiar feature of dimension 3 ). We derive an asymptotic formula for the eigenvalue with smallest modulus for arbitrary perturbations of the metric and present two particular families of Riemannian metrics for which the eigenvalue with smallest modulus can be evaluated explicitly. We also establish a relation between our asymptotic formula and the eta invariant.

Keywords: Dirac operator, spectral asymmetry, zero modes, harmonic spinors, eta invariant

\footnotetext{
a)Electronic mail: R.Downes@ucl.ac.uk; http://www.homepages.ucl.ac.uk/ zcahc37/

b)Electronic mail: M.Levitin@reading.ac.uk http://www.personal.reading.ac.uk/ ny901965/

c)Electronic mail: D.Vassiliev@ucl.ac.uk; http://www.homepages.ucl.ac.uk/ ucahdva/
} 


\section{INTRODUCTION}

Let $M$ be a 3-dimensional connected compact oriented manifold without boundary equipped with a smooth Riemannian metric $g_{\alpha \beta}, \alpha, \beta=1,2,3$ being the tensor indices. Let $W$ be the corresponding massless Dirac operator, see Appendix A in Ref. ${ }^{11}$ for definition. There are two basic examples when the spectrum of $W$ can be calculated explicitly. The first is the unit torus $\mathbb{T}^{3}$ equipped with Euclidean metric. The second is the unit sphere $\mathbb{S}^{3}$ equipped with metric induced by the natural embedding of $\mathbb{S}^{3}$ in Euclidean space $\mathbb{R}^{4}$. In both examples the spectrum turns out to be symmetric about zero, see Appendix B in Ref.11 for details. Physically, this means that in these two examples there is no difference between the properties of the particle (massless neutrino) and antiparticle (massless antineutrino).

As pointed out in Refs. ${ }^{4}[\mathbf{7}$, for a general oriented Riemannian 3-manifold $(M, g)$ there is no reason for the spectrum of the massless Dirac operator $W$ to be symmetric. However, producing explicit examples of spectral asymmetry is a difficult task. To our knowledge, the only explicit example was constructed in Ref.20, with the example based on the idea of choosing a 3-manifold with flat metric but highly nontrivial topology. In our paper we take a different route: we stick with the simplest possible topology (torus) and create spectral asymmetry by perturbing the metric.

Further on in this paper we work on the unit torus $\mathbb{T}^{3}$ parameterized by cyclic coordinates $x^{\alpha}, \alpha=1,2,3$, of period $2 \pi$.

Suppose first that the metric is Euclidean. Then the massless Dirac operator corresponding to the standard spin structure (see formula (A.16) in Ref.11) reads

$$
W=-i\left(\begin{array}{cc}
\frac{\partial}{\partial x^{3}} & \frac{\partial}{\partial x^{1}}-i \frac{\partial}{\partial x^{2}} \\
\frac{\partial}{\partial x^{1}}+i \frac{\partial}{\partial x^{2}} & -\frac{\partial}{\partial x^{3}}
\end{array}\right)
$$

The operator (I.1) admits separation of variables, i.e. one can seek its eigenfunctions in the form $v(x)=u e^{i m_{\alpha} x^{\alpha}}, m \in \mathbb{Z}^{3}, u \in \mathbb{C}^{2}, u \neq 0$, and calculate the eigenvalues and eigenfunctions explicitly. The spectrum of the operator (I.1) is as follows.

- Zero is an eigenvalue of multiplicity two.

- For each $m \in \mathbb{Z}^{3} \backslash\{0\}$ we have the eigenvalue $\|m\|$ and unique (up to rescaling) eigenfunction of the form $u e^{i m_{\alpha} x^{\alpha}}$. 
- For each $m \in \mathbb{Z}^{3} \backslash\{0\}$ we have the eigenvalue $-\|m\|$ and unique (up to rescaling) eigenfunction of the form $u e^{i m_{\alpha} x^{\alpha}}$.

We now perturb the metric, i.e. consider a metric $g_{\alpha \beta}(x ; \epsilon)$ the components of which are smooth functions of coordinates $x^{\alpha}, \alpha=1,2,3$, and small real parameter $\epsilon$, and which satisfies

$$
g_{\alpha \beta}(x ; 0)=\delta_{\alpha \beta} .
$$

One way of establishing spectral asymmetry of the perturbed problem is to compare the asymptotic distribution of large positive eigenvalues and large negative eigenvalues. As explained in Section 10 of Ref $\frac{10}{10}$, for a generic first order differential operator this approach allows one to establish spectral asymmetry. Unfortunately, the massless Dirac operator is very special in that the second asymptotic coefficient of its counting function is zero, see formula (1.23) in Ref $\stackrel{\square 1}{ }$, so in the first two approximations in powers of $\lambda$ its large positive eigenvalues are distributed the same way as its large negative eigenvalues. Therefore, in order to demonstrate spectral asymmetry of the perturbed problem, we will, instead of dealing with large eigenvalues, deal with small eigenvalues.

\section{MAIN RESULT}

Let $W(\epsilon)$ be the massless Dirac operator corresponding to the metric $g_{\alpha \beta}(x ; \epsilon)$. The difficulty with applying standard perturbation techniques to the operator $W(\epsilon)$ is that all its eigenvalues have even multiplicity, this being a consequence of the fact that the massless Dirac operator $W(\epsilon)$ commutes with the antilinear operator of charge conjugation

$$
v=\left(\begin{array}{l}
v_{1} \\
v_{2}
\end{array}\right) \mapsto\left(\begin{array}{c}
-\overline{v_{2}} \\
\overline{v_{1}}
\end{array}\right)=: \mathrm{C}(v),
$$

see Property 3 in Appendix A of Ref.11]. In order to overcome this difficulty we develop in Sections III $\mathrm{V}$ a perturbation theory for the massless Dirac operator which accounts for this charge conjugation symmetry. We show that perturbation-wise the double eigenvalues of the massless Dirac operator can be treated as if they were simple eigenvalues: under perturbation a double eigenvalue remains a double eigenvalue and all the usual formulae apply, with only one minor modification. The minor modification concerns the definition of the pseudoinverse of the unperturbed operator, see formulae (III.8)-(III.12). Namely, 
in the definition of the pseudoinverse we separate out a two-dimensional eigenspace rather than a one-dimensional eigenspace. Here, of course, it is important that we don't have a magnetic field. A magnetic field would split up a double eigenvalue, see Ref.12. The fact that the massless Dirac operator and the charge conjugation operator do not commute in the presence of a magnetic covector potential is well known in theoretical physics: see, for example, formula (2.5) in Ref. 19 .

Given a function $f: \mathbb{T}^{3} \rightarrow \mathbb{C}$, we denote by

$$
\hat{f}(m):=\frac{1}{(2 \pi)^{3}} \int_{\mathbb{T}^{3}} e^{-i m_{\alpha} x^{\alpha}} f(x) d x, \quad m \in \mathbb{Z}^{3},
$$

its Fourier coefficients. Here $d x:=d x^{1} d x^{2} d x^{3}$.

Let $\lambda_{0}(\varepsilon)$ be the eigenvalue of the massless Dirac operator with smallest modulus and let

$$
h_{\alpha \beta}(x):=\left.\frac{\partial g_{\alpha \beta}}{\partial \epsilon}\right|_{\epsilon=0} .
$$

Further on we raise and lower tensor indices using the Euclidean metric, which means that raising or lowering a tensor index doesn't change anything. A repeated tensor index always indicates summation over the values $1,2,3$.

The following theorem is the main result of our paper.

Theorem II.1. We have

$$
\lambda_{0}(\epsilon)=c \epsilon^{2}+O\left(\epsilon^{3}\right) \quad \text { as } \quad \epsilon \rightarrow 0
$$

where the constant $c$ is given by the formula

$$
c=\frac{i}{16} \varepsilon_{\alpha \beta \gamma} \sum_{m \in \mathbb{Z}^{3} \backslash\{0\}}\left(\delta_{\mu \nu}-\frac{m_{\mu} m_{\nu}}{\|m\|^{2}}\right) m_{\alpha} \hat{h}_{\beta \mu}(m) \overline{\hat{h}_{\gamma \nu}(m)} .
$$

Here $\varepsilon_{\alpha \beta \gamma}$ is the totally antisymmetric quantity, $\varepsilon_{123}:=+1$, and the overline stands for complex conjugation.

Theorem II.1 warrants the following remarks.

- If the constant $c$ defined by formula (II.5) is nonzero, then Theorem II.1 tells us that for sufficiently small nonzero $\epsilon$ the spectrum of our massless Dirac operator is asymmetric about zero. 
- Theorem II.1 is in agreement with the established view, see Refs. $\frac{[118}{\text {, }}$, that there are no topological obstructions preventing the shift of the zero eigenvalue of the massless Dirac operator.

- Theorem II.1 is in agreement with the results of Ref. ${ }^{1}$. This paper deals with the Dirac operator in the most general setting. When applied to the case of a compact oriented Riemannian 3-manifold (not necessarily a 3-torus with Euclidean metric) with specified spin structure the results of Ref ${ }^{[1}$ tell us that if zero is an eigenvalue of the Dirac operator, then the metric can be perturbed so that the zero eigenvalue gets shifted. Furthermore, according to Ref ${ }^{2}$, the zero eigenvalue can be shifted by perturbing the metric on an arbitrarily small open set, which is also in agreement with our Theorem II.1.

- Put

$$
\begin{gathered}
L_{\gamma \nu \beta \mu}:=\frac{i \varepsilon_{\alpha \beta \gamma}}{(2 \pi)^{3}} \sum_{m \in \mathbb{Z}^{3} \backslash\{0\}}\left(\delta_{\mu \nu}-\frac{m_{\mu} m_{\nu}}{\|m\|^{2}}\right) m_{\alpha} \int_{\mathbb{T}^{3}} e^{i(x-y)^{\alpha} m_{\alpha}}(\cdot) d y, \\
P_{\gamma \nu \beta \mu}:=\frac{1}{4}\left(L_{\gamma \nu \beta \mu}+L_{\nu \gamma \beta \mu}+L_{\gamma \nu \mu \beta}+L_{\nu \gamma \mu \beta}\right) .
\end{gathered}
$$

This gives us a first order pseudodifferential operator $P$ acting in the vector space of rank two symmetric complex-valued tensor fields, $s_{\beta \mu} \mapsto P_{\gamma \nu \beta \mu} s_{\beta \mu}$. If we equip this vector space with the natural inner product $(r, s):=\int_{\mathbb{T}^{3}} r_{\alpha \beta} \overline{s_{\alpha \beta}} d x$ then it is easy to see that the operator $P$ is formally self-adjoint and formula (II.5) can be rewritten as $c=\frac{1}{128 \pi^{3}}(P h, h)$, where $h$ is defined in accordance with (II.3). This shows that our coefficient $c$ has a nonlocal (global) nature, with the source of the nonlocality being the factor

$$
\delta_{\mu \nu}-\frac{m_{\mu} m_{\nu}}{\|m\|^{2}}
$$

in the symbol of the pseudodifferential operator $P$. In other words, formula (II.5) cannot be rewritten in terms of (linearized) local differential geometric quantities such as the curvature tensor and the Cotton tensor.

- The rank two tensor (II.6) can be identified with a linear map in $\mathbb{R}^{3}, \quad p_{\mu} \mapsto$ $\left(\delta_{\mu \nu}-\frac{m_{\mu} m_{\nu}}{\|m\|^{2}}\right) p_{\nu}$. This linear map is an orthogonal projection: it projects onto the plane orthogonal to the covector (momentum) $m$. 
- Suppose that we are looking at a conformal scaling of the Euclidean metric, $g_{\alpha \beta}(x ; \epsilon)=$ $e^{2 \epsilon \varphi(x)} \delta_{\alpha \beta}$, where $\varphi: \mathbb{T}^{3} \rightarrow \mathbb{R}$. Then $h_{\alpha \beta}(x)=2 \varphi(x) \delta_{\alpha \beta}$ and formula (II.5) becomes

$$
c=\frac{i}{4} \varepsilon_{\alpha \beta \gamma} \sum_{m \in \mathbb{Z}^{3} \backslash\{0\}}\left(\delta_{\mu \nu}-\frac{m_{\mu} m_{\nu}}{\|m\|^{2}}\right) m_{\alpha} \delta_{\beta \mu} \delta_{\gamma \nu}|\hat{\varphi}(m)|^{2} .
$$

The expression in the RHS of (II.7) is zero because the summand in $\sum_{m \in \mathbb{Z}^{3} \backslash\{0\}}$ is odd in $m$. (Another reason why the expression in the RHS of (II.7) is zero is that the summand is symmetric in $\beta, \gamma$.) This agrees with the well-known fact that the zero eigenvalue does not shift under a conformal scaling of the metric, see Theorem 4.3 in Ref. 12 .

- Suppose that we replace the tensor $h_{\alpha \beta}(x)$ by the tensor $h_{\alpha \beta}(-x)$. Then $\hat{h}_{\alpha \beta}(m)$ is replaced by $-\hat{h}_{\alpha \beta}(-m)$ and, introducing a new summation index $n:=-m$ in formula (II.5), we see that the coefficient $c$ changes sign. Physically, this means that formula (II.5) feels the difference between "left" and "right", as one would expect of a formula describing a fermion.

The proof of Theorem II.1 is given in Section VI. In Section VII we treat the special case when the metric $g_{\alpha \beta}(x ; \epsilon)$ is a function of the coordinate $x^{1}$ only. In Sections VIII and IX we present families of metrics for which the eigenvalue $\lambda_{0}(\epsilon)$ can be evaluated explicitly. Finally, in Section $\mathrm{X}$ we examine the eta invariant of our $\epsilon$-dependent massless Dirac operator.

\section{PERTURBATION PROCESS I: PRELIMINARIES}

Let $M$ be a 3-dimensional connected compact oriented manifold without boundary equipped with a smooth Riemannian metric $g_{\alpha \beta}(x), \alpha, \beta=1,2,3$ being the tensor indices and $x=\left(x^{1}, x^{2}, x^{3}\right)$ being local coordinates. The perturbation theory developed in this section and Sections $\mathrm{IV} / \mathrm{V}$ does not assume that the manifold is necessarily a 3 -torus.

We perturb the metric in a smooth manner and denote the perturbed metric by $g_{\alpha \beta}(x ; \epsilon)$, where $\epsilon$ is a small real parameter. Here we assume that $g_{\alpha \beta}(x ; 0)$ is the unperturbed metric described in the previous paragraph.

By $W_{1 / 2}(\epsilon)$ we denote the massless Dirac operator on half-densities corresponding to the metric $g_{\alpha \beta}(x ; \epsilon)$, see Appendix A in Ref ${ }^{[11}$ for details. We choose to work with the massless Dirac operator on half-densities $W_{1 / 2}(\epsilon)$ rather than with the massless Dirac operator $W(\epsilon)$ 
because we do not want our Hilbert space to depend on $\epsilon$. The difference between the operators $W(\epsilon)$ and $W_{1 / 2}(\epsilon)$ is explained in Appendix A of Ref.11]: compare formulae (A.3) and (A.19). The spectra of the operators $W(\epsilon)$ and $W_{1 / 2}(\epsilon)$ are the same.

The operator $W_{1 / 2}(\epsilon)$ is actually not a single operator, but an equivalence class of operators which differ by the transformation

$$
W_{1 / 2}(\epsilon) \mapsto R W_{1 / 2}(\epsilon) R^{*},
$$

where $R(x ; \epsilon)$ is an arbitrary smooth $2 \times 2$ special unitary matrix-function. See Property 4 in Appendix A of Ref ${ }^{[11}$ for a detailed discussion regarding the transformation (III.1), noting that the massless Dirac operator on half-densities $W_{1 / 2}(\epsilon)$ differs from the massless Dirac operator $W(\epsilon)$ only by "scalar" factors on the left and on the right - these "scalar" factors commute with matrix-functions $R(x ; \epsilon)$ and $R^{*}(x ; \epsilon)$. Obviously, the transformation (III.1) does not affect the spectrum. Later on, in Section VI, we will use this gauge degree of freedom to simplify calculations, see formula (VI.11).

The operator $W_{1 / 2}(\epsilon)$ acts on 2-columns $v=\left(\begin{array}{l}v_{1} \\ v_{2}\end{array}\right)$ of complex-valued half-densities. Our Hilbert space is $L^{2}\left(M ; \mathbb{C}^{2}\right)$, which is the vector space of 2-columns of square integrable half-densities equipped with inner product

$$
\langle v, w\rangle:=\int_{M} w^{*} v d x .
$$

The domain of the operator $W_{1 / 2}(\epsilon)$ is $H^{1}\left(M ; \mathbb{C}^{2}\right)$, which is the Sobolev space of 2-columns of half-densities that are square integrable together with their first partial derivatives. It is known that the operator $W_{1 / 2}(\epsilon): H^{1}\left(M ; \mathbb{C}^{2}\right) \rightarrow L^{2}\left(M ; \mathbb{C}^{2}\right)$ is self-adjoint and that it has a discrete spectrum, with eigenvalues accumulating to $+\infty$ and $-\infty$. Note that here neither the Hilbert space nor the domain depend on $\epsilon$. It is also known that the eigenfunctions of the operator $W_{1 / 2}(\epsilon)$ are infinitely smooth.

The antilinear operator of charge conjugation (II.1) maps any element of $L^{2}\left(M ; \mathbb{C}^{2}\right)$ to an element of $L^{2}\left(M ; \mathbb{C}^{2}\right)$ and any element of $H^{1}\left(M ; \mathbb{C}^{2}\right)$ to an element of $H^{1}\left(M ; \mathbb{C}^{2}\right)$. As the massless Dirac operator on half-densities $W_{1 / 2}(\epsilon)$ differs from the massless Dirac operator $W(\epsilon)$ only by real "scalar" factors on the left and on the right, it also commutes with the operator of charge conjugation:

$$
\mathrm{C}\left(W_{1 / 2}(\epsilon) v\right)=W_{1 / 2}(\epsilon) \mathrm{C}(v),
$$


$\forall v \in H^{1}\left(M ; \mathbb{C}^{2}\right)$. Note that the operator of charge conjugation does not itself depend on $\epsilon$. Observe that formulae (II.1) and (III.2) imply the following useful identities:

$$
\begin{gathered}
\mathrm{C}(\mathrm{C}(v))=-v, \\
\langle v, \mathrm{C}(v)\rangle=0, \\
\langle\mathrm{C}(v), \mathrm{C}(w)\rangle=\langle w, v\rangle .
\end{gathered}
$$

Let

$$
W_{1 / 2}(\epsilon)=W_{1 / 2}^{(0)}+\epsilon W_{1 / 2}^{(1)}+\epsilon^{2} W_{1 / 2}^{(2)}+\ldots
$$

be the asymptotic expansion of the partial differential operator $W_{1 / 2}(\epsilon)$ in powers of the small parameter $\epsilon$. Obviously, the operators $W_{1 / 2}^{(k)}, k=0,1,2, \ldots$, are formally self-adjoint first order differential operators which commute with the antilinear operator of charge conjugation (II.1).

Suppose that $\lambda^{(0)}$ is a double eigenvalue of the operator $W_{1 / 2}^{(0)}$. As explained in Appendix A of Ref $\frac{11}{11}$, eigenvalues of the massless Dirac operator have even multiplicity, so a double eigenvalue is the "simplest" eigenvalue one can get.

Remark III.1. The spectrum of the massless Dirac operator on a 3-torus equipped with Euclidean metric was written down explicitly in Section [. Examination of the relevant formulae shows that the only double eigenvalue is the eigenvalue zero as all others have multiplicity greater than or equal to six. However, in this section and Sections $\operatorname{IV} \mathrm{V}$ we do not use the fact that $\lambda^{(0)}=0$.

Let $v^{(0)}$ be a normalized, $\left\|v^{(0)}\right\|=1$, eigenfunction of the operator $W_{1 / 2}^{(0)}$ corresponding to the eigenvalue $\lambda^{(0)}$. Formula III.3 and the fact that $\lambda^{(0)}$ is real imply that $\mathrm{C}\left(v^{(0)}\right)$ is also an eigenfunction of the operator $W_{1 / 2}^{(0)}$ corresponding to the eigenvalue $\lambda^{(0)}$. Formula III.6 implies that $\left\|\mathrm{C}\left(v^{(0)}\right)\right\|=1$, and, moreover, in view of formula (III.5), the eigenfunctions $v^{(0)}$ and $\mathrm{C}\left(v^{(0)}\right)$ are orthogonal.

The argument presented in the previous paragraph shows that, when dealing with a double eigenvalue of the massless Dirac operator, it is sufficient to construct only one eigenfunction: the other one is obtained by charge conjugation. The argument is valid not only for the unperturbed operator $W_{1 / 2}^{(0)}$, but for the perturbed operator $W_{1 / 2}(\epsilon)$ as well, provided that $\epsilon$ is small enough (so that the multiplicity of the eigenvalue does not increase). Hence, in 
the perturbation process described in the next section we shall construct one eigenfunction only.

In the perturbation process that we will describe in the next section we will make use of the pseudoinverse of the unperturbed operator. This operator, which we denote by $Q$, is defined as follows. Consider the problem

$$
\left(W_{1 / 2}^{(0)}-\lambda^{(0)}\right) v=f
$$

where $f \in L^{2}\left(M ; \mathbb{C}^{2}\right)$ is given and $v \in H^{1}\left(M ; \mathbb{C}^{2}\right)$ is to be found. Suppose that $f$ satisfies the conditions

$$
\left\langle f, v^{(0)}\right\rangle=\left\langle f, \mathrm{C}\left(v^{(0)}\right)\right\rangle=0 .
$$

Then the problem (III.8) can be resolved for $v$, however this solution is not unique. We achieve uniqueness by imposing the conditions

$$
\left\langle v, v^{(0)}\right\rangle=\left\langle v, \mathrm{C}\left(v^{(0)}\right)\right\rangle=0
$$

and define $Q$ as the linear operator mapping $f$ to $v$,

$$
Q: f \mapsto v .
$$

Thus, $Q$ is a bounded linear operator acting in the orthogonal complement of the eigenspace of the operator $W_{1 / 2}^{(0)}$ corresponding to the eigenvalue $\lambda^{(0)}$. We extend this operator to the whole Hilbert space $L^{2}\left(M ; \mathbb{C}^{2}\right)$ in accordance with

$$
Q v^{(0)}=Q C\left(v^{(0)}\right)=0 .
$$

It is clear from the above definition that the bounded linear operator $Q$ is self-adjoint and commutes with the antilinear operator of charge conjugation (II.1). Note that our definition of the pseudoinverse $Q$ of the unperturbed operator $W_{1 / 2}^{(0)}-\lambda^{(0)}$ is in agreement with Rellich's, see Chapter 2 Section 2 in Ref. ${ }^{21}$.

Throughout our perturbation process we will have to deal with various formally selfadjoint linear operators which commute with the antilinear operator of charge conjugation (II.1). Such operators possess a special property which is the subject of the following lemma.

Lemma III.2. Let $L: C^{\infty}\left(M ; \mathbb{C}^{2}\right) \rightarrow C^{\infty}\left(M ; \mathbb{C}^{2}\right)$ be a (possibly unbounded) formally selfadjoint linear operator which commutes with the antilinear operator of charge conjugation II.1). Then for any $v \in C^{\infty}\left(M ; \mathbb{C}^{2}\right)$ we have

$$
\langle L v, \mathrm{C}(v)\rangle=0 .
$$


Proof Take arbitrary $v, w \in C^{\infty}\left(M ; \mathbb{C}^{2}\right)$. Using formula III.6) and the fact that $L$ is formally self-adjoint and commutes with $\mathrm{C}$, we get

$$
\langle L \mathrm{C}(w), \mathrm{C}(v)\rangle=\langle\mathrm{C}(L w), \mathrm{C}(v)\rangle=\langle v, L w\rangle=\langle L v, w\rangle .
$$

For $w=\mathrm{C}(v)$ formula (III.14 reads

$$
\langle L \mathrm{C}(\mathrm{C}(v)), \mathrm{C}(v)\rangle=\langle L v, \mathrm{C}(v)\rangle .
$$

But in view of (III.4) formula (III.15) can be rewritten as

$$
-\langle L v, \mathrm{C}(v)\rangle=\langle L v, \mathrm{C}(v)\rangle
$$

which gives us the required identity (III.13.

\section{PERTURBATION PROCESS II: FORMAL PROCEDURE}

We now write down the formal perturbation process. A rigorous justification will be provided in the next section.

Further on in this section as well as in the two following sections (Sections $\mathrm{V}$ and $\mathrm{VI}$ ) we write, for the sake of brevity, $A(\epsilon)=W_{1 / 2}(\epsilon)$ and $A^{(k)}=W_{1 / 2}^{(k)}, k=0,1,2, \ldots$ In this new notation formula (III.7) reads

$$
A(\epsilon)=A^{(0)}+\epsilon A^{(1)}+\epsilon^{2} A^{(2)}+\ldots
$$

We need to solve the eigenvalue problem

$$
A(\epsilon) v(\epsilon)=\lambda(\epsilon) v(\epsilon)
$$

We seek the eigenvalue and eigenfunction of the perturbed operator $A(\epsilon)$ in the form of asymptotic expansions

$$
\begin{aligned}
& \lambda(\epsilon)=\lambda^{(0)}+\epsilon \lambda^{(1)}+\epsilon^{2} \lambda^{(2)}+\ldots, \\
& v(\epsilon)=v^{(0)}+\epsilon v^{(1)}+\epsilon^{2} v^{(2)}+\ldots
\end{aligned}
$$

Note that we do not aim to preserve the normalization of our eigenfunction throughout the perturbation process.

Let us forget for a moment that we are dealing with a double eigenvalue and suppose that our eigenvalue is simple. Then the iterative procedure for the determination of $\lambda^{(k)}$ and 
$v^{(k)}, k=1,2, \ldots$, is well known, see Chapter 2 Section 2 in Ref. ${ }^{21}$. At the $k$ th step we get the equation

$$
\left(A^{(0)}-\lambda^{(0)}\right) v^{(k)}=f^{(k)},
$$

where

$$
f^{(k)}:=F^{(k)} v^{(0)},
$$

and $F^{(k)}$ is some linear operator. The explicit formula for the operator $F^{(k)}$ appearing in equations (IV.5), (IV.6) is written as follows. Put

$$
D(\epsilon):=(B(0)-B(\epsilon))\left(I+\sum_{j=1}^{\infty}[Q(B(0)-B(\epsilon))]^{j}\right),
$$

where $I$ is the identity operator, $B(\epsilon):=A(\epsilon)-\lambda(\epsilon) I$ and the infinite sum is understood as an asymptotic series. The operator $D(\epsilon)$ can be expanded in powers of the small parameter $\epsilon$,

$$
D(\epsilon)=\sum_{k=1}^{\infty} \epsilon^{k} F^{(k)},
$$

giving us the required $F^{(k)}$. The real number $\lambda^{(k)}$ is determined from the condition

$$
\left\langle f^{(k)}, v^{(0)}\right\rangle=0
$$

after which we resolve (IV.5) by setting

$$
v^{(k)}=Q f^{(k)} .
$$

We claim that the above process carries over to the case of a double eigenvalue that we are dealing with. Indeed, the difference between the cases of a simple eigenvalue and a double eigenvalue is that at the $k$ th step of the iterative process in addition to condition (IV.9) we need to satisfy the condition

$$
\left\langle f^{(k)}, \mathrm{C}\left(v^{(0)}\right)\right\rangle=0 .
$$

The structure of the operator (IV.7) is such that it is formally self-adjoint and commutes with the antilinear operator of charge conjugation (II.1), so the operator $F^{(k)}$ defined in accordance with formula (IV.8) has the same properties and, hence, by Lemma III.2, condition (IV.11) is satisfied automatically and the asymptotic process continues as if the eigenvalue were simple. 
We end this section by giving, for future reference, the explicit formulae for the coefficients $\lambda^{(1)}$ and $\lambda^{(2)}$ appearing in the asymptotic expansion (IV.3):

$$
\begin{gathered}
\lambda^{(1)}=\left\langle A^{(1)} v^{(0)}, v^{(0)}\right\rangle, \\
\lambda^{(2)}=\left\langle A^{(2)} v^{(0)}, v^{(0)}\right\rangle-\left\langle\left(A^{(1)}-\lambda^{(1)}\right) Q\left(A^{(1)}-\lambda^{(1)}\right) v^{(0)}, v^{(0)}\right\rangle .
\end{gathered}
$$

\section{PERTURBATION PROCESS III: JUSTIFICATION}

Recall that by $\lambda^{(0)}=\lambda(0)$ we denote a double eigenvalue of the unperturbed operator $A^{(0)}=A(0)$ (the unperturbed massless Dirac operator on half-densities). Let us choose a $\delta>0$ such that $\lambda^{(0)}$ is the only eigenvalue of the operator $A^{(0)}$ on the interval $\left[\lambda^{(0)}-\delta, \lambda^{(0)}+\delta\right]$.

In order to justify our perturbation process we will need the following lemma.

Lemma V.1. For sufficiently small $\epsilon$ the interval

$$
\left(\lambda^{(0)}-\delta, \lambda^{(0)}+\delta\right)
$$

contains exactly one double eigenvalue of the operator $A(\epsilon)$ and no other eigenvalues.

Proof Denote $C_{\delta}:=\left\{\mu \in \mathbb{C}|| \mu-\lambda^{(0)} \mid=\delta\right\}$ (circle in the complex plane) and $D_{\delta}:=$ $\left\{\mu \in \mathbb{C}|| \mu-\lambda^{(0)} \mid<\delta\right\}$ (open disc in the complex plane). Put $R_{\mu}^{(0)}:=\left(A^{(0)}-\mu I\right)^{-1}$. Clearly, for $\mu \in C_{\delta}$ the operator $R_{\mu}^{(0)}$ is well-defined and, moreover, is a bounded operator acting from $L^{2}\left(M ; \mathbb{C}^{2}\right)$ to $H^{1}\left(M ; \mathbb{C}^{2}\right)$. Furthermore, the norm of the operator $R_{\mu}^{(0)}: L^{2}\left(M ; \mathbb{C}^{2}\right) \rightarrow$ $H^{1}\left(M ; \mathbb{C}^{2}\right)$ is bounded uniformly over $\mu \in C_{\delta}$.

Let us now define the operator

$$
R_{\mu}(\epsilon):=\left(I+\sum_{j=1}^{\infty}\left[-R_{\mu}^{(0)}\left(A(\epsilon)-A^{(0)}\right)\right]^{j}\right) R_{\mu}^{(0)},
$$

where $\mu \in C_{\delta}$. The operator $A(\epsilon)-A^{(0)}$ is a bounded operator acting from $H^{1}\left(M ; \mathbb{C}^{2}\right)$ to $L^{2}\left(M ; \mathbb{C}^{2}\right)$ and the norm of the operator $A(\epsilon)-A^{(0)}$ : $H^{1}\left(M ; \mathbb{C}^{2}\right) \rightarrow L^{2}\left(M ; \mathbb{C}^{2}\right)$ tends to zero as $\epsilon$ tends to zero. Hence, the series in $\mathrm{V} .2$ converges for sufficiently small $\epsilon$. Furthermore, it is easy to see that

$$
R_{\mu}(\epsilon) \rightarrow R_{\mu}^{(0)} \quad \text { as } \quad \epsilon \rightarrow 0
$$

in the sense of the operator norm $L^{2}\left(M ; \mathbb{C}^{2}\right) \rightarrow H^{1}\left(M ; \mathbb{C}^{2}\right)$ and this convergence is uniform over $\mu \in C_{\delta}$. 
Acting onto $(\mathrm{V} .2)$ with the operator $A(\epsilon)-\mu I$ we see that $(A(\epsilon)-\mu I) R_{\mu}(\epsilon)=I$, so $R_{\mu}(\epsilon)=(A(\epsilon)-\mu I)^{-1}$. Put

$$
E(\epsilon):=\frac{1}{2 \pi i} \int_{C_{\delta}} R_{\mu}(\epsilon) d \mu .
$$

The operator $E(\epsilon)$ is the orthogonal projection onto the span of eigenvectors of the operator $A(\epsilon)$ corresponding to eigenvalues on the interval $(\mathrm{V} .1)$. In particular, $E(0)=E^{(0)}$ is the orthogonal projection onto the span of eigenvectors of the operator $A^{(0)}$ corresponding to the double eigenvalue $\lambda^{(0)}$.

Formulae (V.3) and (V.4) imply

$$
\left\|E(\epsilon)-E^{(0)}\right\|_{\mathrm{op}} \rightarrow 0 \quad \text { as } \quad \epsilon \rightarrow 0
$$

where $\|\cdot\|_{\text {op }}$ stands for the operator norm in the Banach space of bounded linear operators $L^{2}\left(M ; \mathbb{C}^{2}\right) \rightarrow L^{2}\left(M ; \mathbb{C}^{2}\right)$. Formula $(\mathrm{V} .5$ implies that for sufficiently small $\epsilon$ we have

$$
\left\|E(\epsilon)-E^{(0)}\right\|_{\text {op }}<1 .
$$

Formula (V.6) and the fact that the orthogonal projections $E(\epsilon)$ and $E^{(0)}$ have finite rank imply that $\operatorname{rank} E(\epsilon)=\operatorname{rank} E^{(0)}=2$. Thus, the operator $A(\epsilon)$ has two eigenvalues, counted with multiplicities, on the interval (V.1). We know, see Property 3 in Appendix A of Ref!11, that the eigenvalues of the operator $A(\epsilon)$ have even multiplicity, so we are looking at one double eigenvalue on the interval (V.1).

Let $\lambda(\epsilon)$ be the unique double eigenvalue of the operator $A(\epsilon)$ from the interval (V.1). Denote by $\sigma(\epsilon)$ the spectrum of the operator $A(\epsilon)$ and, for a given $\mu \in \mathbb{R}$, denote $\operatorname{dist}(\mu, \sigma(\epsilon))=$ $\min _{\nu \in \sigma(\epsilon)}|\mu-\nu|$. Obviously, without additional information on $\mu$ and on $\sigma(\epsilon)$ we can only guarantee the inequality

$$
\operatorname{dist}(\mu, \sigma(\epsilon)) \leq|\mu-\lambda(\epsilon)|
$$

Choose an arbitrary natural $k$ and denote

$$
\begin{aligned}
& \tilde{\lambda}(\epsilon)=\lambda^{(0)}+\epsilon \lambda^{(1)}+\epsilon^{2} \lambda^{(2)}+\ldots+\epsilon^{k} \lambda^{(k)}, \\
& \tilde{v}(\epsilon)=v^{(0)}+\epsilon v^{(1)}+\epsilon^{2} v^{(2)}+\ldots+\epsilon^{k} v^{(k)}
\end{aligned}
$$

where the $\lambda^{(j)}$ and $v^{(j)}, j=0,1, \ldots, k$, are taken from IV.3 and IV.4. We have

$$
\|\tilde{v}(\epsilon)\|=1+O(\epsilon)
$$




$$
\|(A(\epsilon)-\tilde{\lambda}(\epsilon)) \tilde{v}(\epsilon)\|=O\left(\epsilon^{k+1}\right),
$$

where $\|\cdot\|$ stands for the $L^{2}\left(M ; \mathbb{C}^{2}\right)$ norm (see (III.2) for inner product). As our operator $A(\epsilon)$ is self-adjoint, formulae (V.10) and (V.11) imply

$$
\operatorname{dist}(\tilde{\lambda}(\epsilon), \sigma(\epsilon)) \leq \frac{\|(A(\epsilon)-\tilde{\lambda}(\epsilon)) \tilde{v}(\epsilon)\|}{\|\tilde{v}(\epsilon)\|}=O\left(\epsilon^{k+1}\right) .
$$

Formulae $(\mathrm{V} .8)$ and $(\mathrm{V} .12)$ and Lemma $\mathrm{V} .1$ imply that for sufficiently small $\epsilon$,

$$
\operatorname{dist}(\tilde{\lambda}(\epsilon), \sigma(\epsilon))=|\tilde{\lambda}(\epsilon)-\lambda(\epsilon)|
$$

compare with V.7). Combining formulae $\mathrm{V} .12$ and $\mathrm{V} .13$, we get $\lambda(\epsilon)=\tilde{\lambda}(\epsilon)+O\left(\epsilon^{k+1}\right)$. This completes the justification of our perturbation process.

\section{PROOF OF THEOREM II.1}

The unperturbed massless Dirac operator on half-densities, which we denote by $A^{(0)}$, is given by the expression in the RHS of (I.1). The unperturbed eigenvalue, $\lambda^{(0)}$, is zero and the corresponding normalized eigenfunction is

$$
v^{(0)}=\frac{1}{(2 \pi)^{3 / 2}}\left(\begin{array}{l}
1 \\
0
\end{array}\right) .
$$

The pseudoinverse $Q$ of the operator $A^{(0)}$ is given by the formula

$$
\begin{aligned}
Q=\frac{1}{(2 \pi)^{3}} & \sum_{m \in \mathbb{Z}^{3} \backslash\{0\}} e^{i m_{\alpha} x^{\alpha}}\left(\begin{array}{cc}
m_{3} & m_{1}-i m_{2} \\
m_{1}+i m_{2} & -m_{3}
\end{array}\right)^{-1} \int_{\mathbb{T}^{3}} e^{-i m_{\alpha} y^{\alpha}}(\cdot) d y \\
=\frac{1}{(2 \pi)^{3}} & \sum_{m \in \mathbb{Z}^{3} \backslash\{0\}} \frac{e^{i m_{\alpha} x^{\alpha}}}{\|m\|^{2}}\left(\begin{array}{cc}
m_{3} & m_{1}-i m_{2} \\
m_{1}+i m_{2} & -m_{3}
\end{array}\right) \int_{\mathbb{T}^{3}} e^{-i m_{\alpha} y^{\alpha}}(\cdot) d y,
\end{aligned}
$$

where $d y:=d y^{1} d y^{2} d y^{3}$. The operator $(\mathrm{VI} .2)$ is a self-adjoint pseudodifferential operator of order -1 .

We have

$$
\lambda(\epsilon)=\epsilon \lambda^{(1)}+\epsilon^{2} \lambda^{(2)}+O\left(\epsilon^{3}\right),
$$

where the coefficients $\lambda^{(1)}$ and $\lambda^{(2)}$ are given by formulae (IV.12) and IV.13) respectively. Thus, in order to prove Theorem II.1 we need to write down explicitly the differential 
operators $A^{(1)}$ and $A^{(2)}$ appearing in the asymptotic expansion of the perturbed massless Dirac operator on half-densities,

$$
A(\epsilon)=A^{(0)}+\epsilon A^{(1)}+\epsilon^{2} A^{(2)}+O\left(\epsilon^{3}\right) .
$$

In what follows we use terminology from microlocal analysis. In particular, we use the notions of the principal and subprincipal symbols of a differential operator, see subsection 2.1.3 in Ref. ${ }^{22}$ for details.

Let $L$ be a first order $2 \times 2$ matrix differential operator. We denote its principal and subprincipal symbols by $L_{1}(x, \xi)$ and $L_{\text {sub }}(x)$ respectively. Here $\xi=\left(\xi_{1}, \xi_{2}, \xi_{3}\right)$ is the variable dual to the position variable $x$; in physics literature the $\xi$ would be referred to as momentum. The subscript in $L_{1}(x, \xi)$ indicates the degree of homogeneity in $\xi$.

A first order differential operator $L$ is completely determined by its principal and subprincipal symbols. Indeed, the principal symbol has the form

$$
L_{1}(x, \xi)=M^{(\alpha)}(x) \xi_{\alpha}
$$

where $M^{(\alpha)}(x)$ are matrix-functions depending only on the position variable $x$. It is easy to see that the differential operator $L$ is given by the formula

$$
L=-\frac{i}{2} M^{(\alpha)}(x) \frac{\partial}{\partial x^{\alpha}}-\frac{i}{2} \frac{\partial}{\partial x^{\alpha}} M^{(\alpha)}(x)+L_{\mathrm{sub}}(x) .
$$

Given a first order differential operator $L$, let us consider the expression $\left\langle L v^{(0)}, v^{(0)}\right\rangle$, where $v^{(0)}$ is the constant column (VI.1) and angular brackets indicate the inner product (III.2). Examination of formula (VI.6) shows that

$$
\left\langle L v^{(0)}, v^{(0)}\right\rangle=\left\langle L_{\mathrm{sub}} v^{(0)}, v^{(0)}\right\rangle
$$

because the terms coming from the principal symbol integrate to zero. Consequently formulae (IV.12) and (IV.13) simplify and now read

$$
\begin{gathered}
\lambda^{(1)}=\left\langle A_{\mathrm{sub}}^{(1)} v^{(0)}, v^{(0)}\right\rangle \\
\lambda^{(2)}=\left\langle A_{\mathrm{sub}}^{(2)} v^{(0)}, v^{(0)}\right\rangle-\left\langle\left(A^{(1)}-\lambda^{(1)}\right) Q\left(A^{(1)}-\lambda^{(1)}\right) v^{(0)}, v^{(0)}\right\rangle .
\end{gathered}
$$

We see that for the purpose of proving Theorem II.1 we do not need to know the full operator $A^{(2)}$, only its subprincipal symbol $A_{\text {sub }}^{(2)}$. 
In order to write down explicitly the massless Dirac operator on half-densities $A(\epsilon)$ we need the concepts of frame and coframe. The differential geometric definition of coframe was given in Section 3 of Ref.11. However, as in the current paper we are working in a specified coordinate system, we can adopt a somewhat simpler approach. For the purposes of the current paper a coframe is a smooth real-valued matrix-function $e^{j}{ }_{\alpha}(x ; \epsilon), j, \alpha=1,2,3$, satisfying the conditions

$$
\begin{gathered}
g_{\alpha \beta}(x ; \epsilon)=\delta_{j k} e^{j}{ }_{\alpha}(x ; \epsilon) e^{k}{ }_{\beta}(x ; \epsilon), \\
e^{j}{ }_{\alpha}(x ; 0)=\delta^{j}{ }_{\alpha} .
\end{gathered}
$$

Here and further on when dealing with matrix-functions we use the convention that the first index (subscript or superscript) enumerates the rows and the second index (subscript or superscript) enumerates the columns. Say, in matrix notation the RHS of (VI.9) reads as "product of coframe transposed and coframe".

Note that the reason we imposed condition VI.10) is so that our unperturbed operator has the form (I.1). See also formula (III.1) and associated discussion.

For a given metric $g_{\alpha \beta}(x ; \epsilon)$ the coframe $e_{\alpha}^{j}(x ; \epsilon)$ is not defined uniquely. We can multiply the matrix-function $e^{j}{ }_{\alpha}(x ; \epsilon)$ from the left by an arbitrary smooth $3 \times 3$ special orthogonal matrix-function $O(x ; \epsilon)$ satisfying the condition $O(x ; 0)=I$, with $I$ denoting the $3 \times 3$ identity matrix. This will give us a new coframe satisfying the defining relations (VI.9) and VI.10. As explained in Appendix A of Ref. 11 , this freedom in the choice of coframe is a gauge degree of freedom which does not affect the spectrum. In the current section we specify the gauge by requiring the matrix-function $e^{j}{ }_{\alpha}(x ; \epsilon)$ to be symmetric,

$$
e^{j}{ }_{\alpha}(x ; \epsilon)=e^{\alpha}{ }_{j}(x ; \epsilon) .
$$

Condition (VI.11) makes sense because we are working in a specified coordinate system. Looking ahead, let us point out the main advantage of the symmetric gauge (VI.11): the asymptotic expansion of the subprincipal symbol of the massless Dirac operator on halfdensities in powers of $\epsilon$ starts with a quadratic term and, moreover, the coefficient at $\epsilon^{2}$ has an especially simple structure, see formulae (VI.16) and (VI.19).

In matrix notation condition (VI.9) now reads "the symmetric positive matrix $g_{\alpha \beta}(x ; \epsilon)$ is the square of the symmetric matrix $e^{j}{ }_{\alpha}(x ; \epsilon)$ ". Conversely, the symmetric matrix $e^{j}{ }_{\alpha}(x ; \epsilon)$ is the square root of the symmetric positive matrix $g_{\alpha \beta}(x ; \epsilon)$. We choose the branch of the square root so that the matrix $e^{j}{ }_{\alpha}(x ; \epsilon)$ is positive. 
According to formulae $($ I.2) and (II.3) we have

$$
g_{\alpha \beta}(x ; \epsilon)=\delta_{\alpha \beta}+\epsilon h_{\alpha \beta}(x)+O\left(\epsilon^{2}\right),
$$

hence, by Taylor's formula for $\sqrt{1+z}$,

$$
e^{j}{ }_{\alpha}(x ; \epsilon)=\delta^{j}{ }_{\alpha}+\frac{\epsilon}{2} h^{j}{ }_{\alpha}(x)+O\left(\epsilon^{2}\right) .
$$

Here we follow the convention introduced in Section III we raise and lower indices in $h$ using the Euclidean metric, which means that raising or lowering an index doesn't change anything. We also swap, when needed, tensor (Greek) indices for frame (Latin) indices, which is acceptable because we are working in a specified coordinate system.

The frame is the smooth real-valued matrix-function $e_{j}{ }^{\alpha}(x ; \epsilon), j, \alpha=1,2,3$, defined by the system of linear algebraic equations

$$
e_{j}^{\alpha}(x ; \epsilon) e_{\alpha}^{k}(x ; \epsilon)=\delta_{j}^{k} .
$$

Note the position of indices in formula (VI.14). In matrix notation formula (VI.14) reads as "the frame is the transpose of the inverse of the coframe". As we chose our coframe to be symmetric, our frame is also symmetric and is simply the inverse of the coframe. Formula (VI.13) and Taylor's formula for $(1+z)^{-1}$ imply

$$
e_{j}^{\alpha}(x ; \epsilon)=\delta_{j}{ }^{\alpha}-\frac{\epsilon}{2} h_{j}^{\alpha}(x)+O\left(\epsilon^{2}\right) .
$$

According to formulae (6.1), (3.5) and (8.1) from Ref 11 the subprincipal symbol of the massless Dirac operator on half-densities is

$$
A_{\mathrm{sub}}(x ; \epsilon)=\frac{3}{4}\left(* T^{\mathrm{ax}}(x ; \epsilon)\right) I
$$

where $I$ is the $2 \times 2$ identity matrix and $* T^{\mathrm{ax}}(x ; \epsilon)$ is the scalar function

$$
\begin{aligned}
* T^{\mathrm{ax}}=\frac{\delta_{k l}}{3} \sqrt{\operatorname{det} g^{\alpha \beta}}\left[e^{k}{ }_{1} \partial e^{l}{ }_{3} / \partial x^{2}\right. & +e^{k}{ }_{2} \partial e_{1}^{l} / \partial x^{3}+e^{k}{ }_{3} \partial e_{2}^{l} / \partial x^{1} \\
& \left.-e^{k}{ }_{1} \partial e^{l}{ }_{2} / \partial x^{3}-e^{k}{ }_{2} \partial e_{3}^{l} / \partial x^{1}-e^{k}{ }_{3} \partial e_{1}^{l} / \partial x^{2}\right] .
\end{aligned}
$$

Note that formula VI.12 implies $g^{\alpha \beta}(x ; \epsilon)=\delta_{\alpha \beta}-\epsilon h^{\alpha \beta}(x)+O\left(\epsilon^{2}\right)$, which, in turn, gives us

$$
\sqrt{\operatorname{det} g^{\alpha \beta}(x ; \epsilon)}=1-\frac{\epsilon}{2} \operatorname{tr} h(x)+O\left(\epsilon^{2}\right) .
$$


Substituting formulae (VI.18) and (VI.13) into (VI.17) and using the symmetry condition (VI.11), we get

$$
\begin{aligned}
& * T^{\operatorname{ax}}(x ; \epsilon)= \\
& \begin{aligned}
\frac{\epsilon^{2} \delta^{k l}}{12}\left[h_{k 1} \frac{\partial h_{l 3}}{\partial x^{2}}+h_{k 2} \frac{\partial h_{l 1}}{\partial x^{3}}+h_{k 3} \frac{\partial h_{l 2}}{\partial x^{1}}-h_{k 1} \frac{\partial h_{l 2}}{\partial x^{3}}\right. & \left.-h_{k 2} \frac{\partial h_{l 3}}{\partial x^{1}}-h_{k 3} \frac{\partial h_{l 1}}{\partial x^{2}}\right]+O\left(\epsilon^{3}\right) \\
& =-\frac{\epsilon^{2}}{12} \varepsilon_{\beta \gamma \delta} h_{\alpha \beta} \frac{\partial h_{\alpha \gamma}}{\partial x^{\delta}}+O\left(\epsilon^{3}\right) .
\end{aligned}
\end{aligned}
$$

Formulae (VI.16) and (VI.19) imply

$$
\begin{gathered}
A_{\mathrm{sub}}^{(1)}(x)=0, \\
A_{\mathrm{sub}}^{(2)}(x)=-\frac{1}{16} \varepsilon_{\beta \gamma \delta} h_{\alpha \beta} \frac{\partial h_{\alpha \gamma}}{\partial x^{\delta}} I .
\end{gathered}
$$

Substituting (VI.20) into (VI.7) we get $\lambda^{(1)}=0$. Formulae $($ VI.3) and (VI.8) now simplify and read

$$
\begin{gathered}
\lambda(\epsilon)=c \epsilon^{2}+O\left(\epsilon^{3}\right), \\
c=\lambda^{(2)}=\left\langle A_{\mathrm{sub}}^{(2)} v^{(0)}, v^{(0)}\right\rangle-\left\langle A^{(1)} Q A^{(1)} v^{(0)}, v^{(0)}\right\rangle .
\end{gathered}
$$

In order to complete our calculation we now need only to write down the principal symbol $A_{1}^{(1)}(x, \xi)$ of the differential operator $A^{(1)}$.

According to formulae (A.1)-(A.3) and (A.19) from Ref.11 the principal symbol of the massless Dirac operator on half-densities is

$$
A_{1}(x, \xi ; \epsilon)=\left(\begin{array}{cc}
e_{3}{ }^{\alpha} & e_{1}^{\alpha}-i e_{2}^{\alpha} \\
e_{1}^{\alpha}+i e_{2}{ }^{\alpha} & -e_{3}{ }^{\alpha}
\end{array}\right) \xi_{\alpha} .
$$

Formulae (VI.24) and (VI.15) imply

$$
A_{1}^{(1)}(x, \xi)=-\frac{1}{2}\left(\begin{array}{cc}
h_{3}{ }^{\alpha} & h_{1}{ }^{\alpha}-i h_{2}{ }^{\alpha} \\
h_{1}{ }^{\alpha}+i h_{2}{ }^{\alpha} & -h_{3}{ }^{\alpha}
\end{array}\right) \xi_{\alpha} .
$$

Formulae (VI.5), (VI.6), (VI.20) and (VI.25) allow us to write down the differential operator $A^{(1)}$ explicitly:

$$
A^{(1)}=\frac{i}{4}\left(\begin{array}{cc}
h_{3}{ }^{\alpha} & h_{1}{ }^{\alpha}-i h_{2}{ }^{\alpha} \\
h_{1}{ }^{\alpha}+i h_{2}{ }^{\alpha} & -h_{3}{ }^{\alpha}
\end{array}\right) \frac{\partial}{\partial x^{\alpha}}+\frac{i}{4} \frac{\partial}{\partial x^{\alpha}}\left(\begin{array}{cc}
h_{3}{ }^{\alpha} & h_{1}{ }^{\alpha}-i h_{2}{ }^{\alpha} \\
h_{1}{ }^{\alpha}+i h_{2}{ }^{\alpha} & -h_{3}{ }^{\alpha}
\end{array}\right) .
$$

Substituting formulae (VI.1), (VI.2), (VI.21) and (VI.26) into (VI.23) we arrive at (II.5) . This completes the proof of Theorem II.1. 


\section{AXISYMMETRIC CASE}

An important special case is when the metric $g_{\alpha \beta}(x ; \epsilon)$ is a function of the coordinate $x^{1}$ only. In this case one can choose the coframe and frame so that they depend on the coordinate $x^{1}$ only and seek eigenfunctions in the form

$$
v(x)=u\left(x^{1}\right) e^{i\left(m_{2} x^{2}+m_{3} x^{3}\right)}, \quad m_{2}, m_{3} \in \mathbb{Z} .
$$

We get separation of variables, i.e. the original eigenvalue problem for a partial differential operator reduces to an eigenvalue problem for an ordinary differential operator depending on the integers $m_{2}$ and $m_{3}$ as parameters. As we know the spectrum of the unperturbed operator (see Section I) and as the eigenvalues of the original partial differential operator depend on the small parameter $\epsilon$ continuously, the eigenvalue with smallest modulus will come from the ordinary differential operator with $m_{2}=m_{3}=0$. We call the case $m_{2}=m_{3}=0$ the axisymmetric case.

The axisymmetric massless Dirac operator on half-densities reads

$$
\begin{array}{r}
W_{1 / 2}(\epsilon)= \\
-\frac{i}{2}\left(\begin{array}{rr}
e_{3}{ }^{1} & e_{1}{ }^{1}-i e_{2}{ }^{1} \\
e_{1}{ }^{1}+i e_{2}{ }^{1} & -e_{3}{ }^{1}
\end{array}\right) \frac{d}{d x^{1}}-\frac{i}{2} \frac{d}{d x^{1}}\left(\begin{array}{cc}
e_{3}{ }^{1} & e_{1}{ }^{1}-i e_{2}{ }^{1} \\
e_{1}{ }^{1}+i e_{2}{ }^{1} & -e_{3}{ }^{1}
\end{array}\right) \\
+\frac{\delta_{j k}}{4 \sqrt{\operatorname{det} g_{\alpha \beta}}}\left[e^{j}{ }_{3}\left(\frac{d e^{k}{ }_{2}}{d x^{1}}\right)-e^{j}{ }_{2}\left(\frac{d e^{k}}{d x^{1}}\right)\right] I,
\end{array}
$$

where $I$ is the $2 \times 2$ identity matrix and

$$
\sqrt{\operatorname{det} g_{\alpha \beta}}=\frac{1}{\sqrt{\operatorname{det} g^{\alpha \beta}}}=\operatorname{det} e^{j}{ }_{\alpha}=\frac{1}{\operatorname{det} e_{j}{ }^{\alpha}} .
$$

Here $e_{\alpha}^{j}\left(x^{1} ; \epsilon\right)$ and $e_{j}{ }^{\alpha}\left(x^{1} ; \epsilon\right)$ are the coframe and frame defined in accordance with formulae VI.9), (VI.10) and VI.14).

Of course, for a given metric $g_{\alpha \beta}\left(x^{1} ; \epsilon\right)$ the coframe $e^{j}{ }_{\alpha}\left(x^{1} ; \epsilon\right)$ and frame $e_{j}{ }^{\alpha}\left(x^{1} ; \epsilon\right)$ are not defined uniquely. We can multiply the matrix-functions $e^{j}{ }_{\alpha}\left(x^{1} ; \epsilon\right)$ and $e_{j}{ }^{\alpha}\left(x^{1} ; \epsilon\right)$ from the left by an arbitrary smooth $3 \times 3$ special orthogonal matrix-function $O\left(x^{1} ; \epsilon\right)$ satisfying the condition $O\left(x^{1} ; 0\right)=I$, with $I$ denoting the $3 \times 3$ identity matrix. This will give us a new coframe and a new frame satisfying the defining relations (VI.9), (VI.10) and (VI.14). Note that in writing down formula (VII.1) we did not assume a particular choice of gauge, compare with VI.11. 
In the axisymmetric case formula (II.5) also simplifies and reads now

$$
c=-\frac{1}{8} \sum_{m_{1} \in \mathbb{N}} m_{1} \operatorname{tr}\left[\left(\begin{array}{ll}
\hat{h}_{22} & \hat{h}_{23} \\
\hat{h}_{32} & \hat{h}_{33}
\end{array}\right)\left(\begin{array}{cc}
0 & -i \\
i & 0
\end{array}\right)\left(\begin{array}{ll}
\hat{h}_{22} & \hat{h}_{23} \\
\hat{h}_{32} & \hat{h}_{33}
\end{array}\right)^{*}\right] \text {, }
$$

where $\hat{h}_{\alpha \beta}=\hat{h}_{\alpha \beta}\left(m_{1}\right)$ and the star stands for Hermitian conjugation.

The next two sections deal with explicit examples of the axisymmetric case just treated.

\section{EXAMPLE OF QUADRATIC DEPENDENCE ON $\epsilon$}

Consider the metric

$$
\begin{aligned}
g_{\alpha \beta}\left(x^{1} ; \epsilon\right) d x^{\alpha} d x^{\beta}=\left[d x^{1}\right]^{2}+\left[\left(1+\epsilon\left(\cos x^{1}\right)\right) d x^{2}+\epsilon\left(\sin x^{1}\right) d x^{3}\right]^{2} \\
+\left[\epsilon\left(\sin x^{1}\right) d x^{2}+\left(1-\epsilon\left(\cos x^{1}\right)\right) d x^{3}\right]^{2} .
\end{aligned}
$$

Then

$$
e_{\alpha}^{j}\left(x^{1} ; \epsilon\right)=\delta^{j}{ }_{\alpha}+\epsilon\left(\begin{array}{ccc}
0 & 0 & 0 \\
0 & \cos x^{1} & \sin x^{1} \\
0 & \sin x^{1} & -\cos x^{1}
\end{array}\right)
$$

is a coframe associated with the metric (VIII.1), see formulae (VI.9), (VI.10), and

$$
e_{j}^{\alpha}\left(x^{1} ; \epsilon\right)=\left(\begin{array}{ccc}
1 & 0 & 0 \\
0 & \frac{1-\epsilon \cos x^{1}}{1-\epsilon^{2}} & -\frac{\epsilon \sin x^{1}}{1-\epsilon^{2}} \\
0 & -\frac{\epsilon \sin x^{1}}{1-\epsilon^{2}} & \frac{1+\epsilon \cos x^{1}}{1-\epsilon^{2}}
\end{array}\right)
$$

is the corresponding frame, see formula (VI.14). Note that in writing formula (VIII.3) we used the fact that

$$
\operatorname{det} e^{j}{ }_{\alpha}(x ; \epsilon)=1-\epsilon^{2}=\sqrt{\operatorname{det} g_{\alpha \beta}} .
$$

Substituting formulae (VIII.2)-(VIII.4) into (VII.1) we get

$$
W(\epsilon)=-i\left(\begin{array}{ll}
0 & 1 \\
1 & 0
\end{array}\right) \frac{d}{d x^{1}}-\frac{\epsilon^{2}}{2\left(1-\epsilon^{2}\right)} I .
$$

Note that in the LHS we dropped the subscript 1/2: as the Riemannian density is constant, see (VIII.4), there is no need to distinguish the massless Dirac operator $W(\epsilon)$ and the massless Dirac operator on half-densities $W_{1 / 2}(\epsilon)$. 
It is easy to see that the eigenvalues of the ordinary differential operator (VIII.5) subject to the (boundary) condition of $2 \pi$-periodicity are

$$
\lambda_{n}(\epsilon)=n-\frac{\epsilon^{2}}{2\left(1-\epsilon^{2}\right)}, \quad n \in \mathbb{Z},
$$

and that all eigenvalues have multiplicity two. In particular, the eigenvalue with smallest modulus is

$$
\lambda_{0}(\epsilon)=-\frac{\epsilon^{2}}{2\left(1-\epsilon^{2}\right)}=-\frac{\epsilon^{2}}{2}+O\left(\varepsilon^{4}\right) \quad \text { as } \quad \varepsilon \rightarrow 0 .
$$

Let us now test Theorem II.1 by comparing the asymptotic formula from this theorem with formula (VIII.6). Substituting (VIII.1) into (II.3) we get

$$
h_{\alpha \beta}\left(x^{1}\right)=2\left(\begin{array}{ccc}
0 & 0 & 0 \\
0 & \cos x^{1} & \sin x^{1} \\
0 & \sin x^{1} & -\cos x^{1}
\end{array}\right) \text {. }
$$

Application of the Fourier transform (II.2) gives us

$$
\hat{h}_{\alpha \beta}\left(m_{1}\right)=\left\{\begin{array}{ccc}
\left(\begin{array}{ccc}
0 & 0 & 0 \\
0 & 1 & -i \\
0 & -i & -1
\end{array}\right) & \text { for } m_{1}=1, \\
0 & \text { for } m_{1}=2,3, \ldots
\end{array}\right.
$$

Substituting VIII.7) into VII.2 we get $c=-\frac{1}{2}$, in agreement with VIII.6).

\section{EXAMPLE OF QUARTIC DEPENDENCE ON $\epsilon$}

Consider the metric

$$
g_{\alpha \beta}\left(x^{1} ; \epsilon\right) d x^{\alpha} d x^{\beta}=\left[d x^{1}+\epsilon\left(\cos x^{1}\right) d x^{2}+\epsilon\left(\sin x^{1}\right) d x^{3}\right]^{2}+\left[d x^{2}\right]^{2}+\left[d x^{3}\right]^{2}
$$

Then

$$
e^{j}{ }_{\alpha}\left(x^{1} ; \epsilon\right)=\delta^{j}{ }_{\alpha}+\epsilon\left(\begin{array}{ccc}
0 & \cos x^{1} & \sin x^{1} \\
0 & 0 & 0 \\
0 & 0 & 0
\end{array}\right)
$$


is a coframe associated with the metric (IX.1), see formulae (VI.9), (VI.10), and

$$
e_{j}^{\alpha}\left(x^{1} ; \epsilon\right)=\delta_{j}^{\alpha}-\epsilon\left(\begin{array}{ccc}
0 & 0 & 0 \\
\cos x^{1} & 0 & 0 \\
\sin x^{1} & 0 & 0
\end{array}\right)
$$

is the corresponding frame, see formula (VI.14). Note that in writing formula (IX.3) we used the fact that

$$
\operatorname{det} e^{j}(x ; \epsilon)=1=\sqrt{\operatorname{det} g_{\alpha \beta}} .
$$

Substituting formulae (IX.2)-(IX.4) into (VII.1) we get

$$
\begin{aligned}
W(\epsilon)=-i\left(\begin{array}{ll}
0 & 1 \\
1 & 0
\end{array}\right) & \frac{d}{d x^{1}}-\frac{\epsilon^{2}}{4} I \\
& +\frac{i \epsilon}{2}\left(\begin{array}{cc}
\sin x^{1} & -i \cos x^{1} \\
i \cos x^{1} & -\sin x^{1}
\end{array}\right) \frac{d}{d x^{1}}+\frac{i \epsilon}{2} \frac{d}{d x^{1}}\left(\begin{array}{cc}
\sin x^{1} & -i \cos x^{1} \\
i \cos x^{1} & -\sin x^{1}
\end{array}\right) .
\end{aligned}
$$

Note that in the LHS we dropped the subscript 1/2: as the Riemannian density is constant, see (IX.4), there is no need to distinguish the massless Dirac operator $W(\epsilon)$ and the massless Dirac operator on half-densities $W_{1 / 2}(\epsilon)$.

We shall now rewrite the ordinary differential operator (IX.5) in a somewhat more convenient form. To this end, let us introduce the special unitary matrix

$$
R:=\frac{1}{\sqrt{2}}\left(\begin{array}{cc}
1 & 1 \\
-1 & 1
\end{array}\right)
$$

and put

$$
\tilde{W}(\epsilon):=R W(\epsilon) R^{*},
$$

compare with formula III.1. Clearly, the operator $\tilde{W}(\epsilon)$ has the same spectrum as the operator $W(\epsilon)$. Substituting (IX.5) and (IX.6) into (IX.7) we arrive at the following explicit formula for the ordinary differential operator $\tilde{W}(\epsilon)$ :

$$
\begin{aligned}
\tilde{W}(\epsilon)=-i\left(\begin{array}{cc}
1 & 0 \\
0 & -1
\end{array}\right) \frac{d}{d x^{1}}- & \frac{\epsilon^{2}}{4} I \\
& +\frac{i \epsilon}{2}\left(\begin{array}{cc}
0 & -i e^{-i x^{1}} \\
i e^{i x^{1}} & 0
\end{array}\right) \frac{d}{d x^{1}}+\frac{i \epsilon}{2} \frac{d}{d x^{1}}\left(\begin{array}{cc}
0 & -i e^{-i x^{1}} \\
i e^{i x^{1}} & 0
\end{array}\right) .
\end{aligned}
$$


The coefficients of the ordinary differential operator (IX.8) are trigonometric polynomials and one would not normally expect the eigenfunctions to be trigonometric polynomials. However, the operator (IX.8) has a special structure which ensures that the eigenfunctions are trigonometric polynomials. Namely, put

$$
\begin{array}{ll}
\lambda_{n}(\epsilon)=-\frac{1}{2}-\frac{\epsilon^{2}}{4}+\sqrt{1+\epsilon^{2}}\left(n+\frac{1}{2}\right), & n \in \mathbb{Z}, \\
v^{(n)}\left(x^{1} ; \epsilon\right)=\left(\begin{array}{c}
\left(1+\sqrt{1+\epsilon^{2}}\right) e^{i n x^{1}} \\
-i \epsilon e^{i(n+1) x^{1}}
\end{array}\right), & n \in \mathbb{Z} .
\end{array}
$$

It is easy to see that the column-functions (IX.10) are eigenfunctions of the operator (IX.8) corresponding to eigenvalues (IX.9). Moreover, it is easy to see that the charge conjugates, $\mathrm{C}\left(v^{(n)}\left(x^{1} ; \epsilon\right)\right)$, of the column-functions IX.10 are eigenfunctions of the operator IX.8) corresponding to the same eigenvalues (IX.9). This means that the numbers (IX.9) are eigenvalues of the operator IX.8) of multiplicity at least two. Finally, it is easy to see that

$$
\begin{aligned}
\operatorname{span}\left\{v^{(n)}\left(x^{1} ; \epsilon\right), \mathrm{C}\left(v^{(n)}\left(x^{1} ; \epsilon\right)\right) \mid n\right. & \in \mathbb{Z}\} \\
& =\operatorname{span}\left\{v^{(n)}\left(x^{1} ; 0\right), \mathrm{C}\left(v^{(n)}\left(x^{1} ; 0\right)\right) \mid n \in \mathbb{Z}\right\},
\end{aligned}
$$

where span $S$ denotes the linear span, i.e. set of all finite linear combinations of elements of a given set $S$. Formula (IX.11) implies that we haven't missed any eigenvalues, that is, that the list (IX.9) contains all the eigenvalues of the operator (IX.8) and that each of these eigenvalues has multiplicity two.

Remark IX.1. We do not fully understand the underlying reasons why the axisymmetric massless Dirac operator corresponding to the metric (IX.1) admits an explicit evaluation of the eigenvalues and eigenfunctions. Somehow, this particular Dirac operator has properties similar to those of an integrable system.

The eigenvalue (IX.9) with smallest modulus is

$$
\lambda_{0}(\epsilon)=\frac{2 \sqrt{1+\epsilon^{2}}-2-\epsilon^{2}}{4}=-\frac{\epsilon^{4}}{16}+O\left(\epsilon^{6}\right) \quad \text { as } \quad \varepsilon \rightarrow 0 .
$$

Let us now test Theorem II.1 by comparing the asymptotic formula from this theorem with formula (IX.12). Substituting IX.1) into II.3) we get

$$
h_{\alpha \beta}\left(x^{1}\right)=\left(\begin{array}{ccc}
0 & \cos x^{1} & \sin x^{1} \\
\cos x^{1} & 0 & 0 \\
\sin x^{1} & 0 & 0
\end{array}\right) .
$$


Application of the Fourier transform (II.2) gives us

$$
\hat{h}_{\alpha \beta}\left(m_{1}\right)=\left\{\begin{array}{ccc}
\left(\begin{array}{ccc}
0 & \frac{1}{2} & -\frac{i}{2} \\
\frac{1}{2} & 0 & 0 \\
-\frac{i}{2} & 0 & 0
\end{array}\right) & \text { for } m_{1}=1, \\
& & \\
0 & \text { for } m_{1}=2,3, \ldots
\end{array}\right.
$$

Substituting (IX.13) into (VII.2) we get $c=0$, in agreement with IX.12).

\section{THE ETA INVARIANT}

Let $H$ be a first order self-adjoint elliptic $m \times m$ matrix classical pseudodifferential operator acting on $m$-columns of complex-valued half-densities over a compact $n$-dimensional manifold $M$ without boundary. Here ellipticity is understood as the nonvanishing of the determinant of the principal symbol of $H$, see Ref.10. The eta function of $H$ is defined as

$$
\eta_{H}(s):=\sum \frac{\operatorname{sign} \lambda}{|\lambda|^{s}}
$$

where summation is carried out over all nonzero eigenvalues $\lambda$ of $H$, and $s \in \mathbb{C}$ is the independent variable. Asymptotic formulae for the counting function imply that the series (X.1) converges absolutely for $\operatorname{Re} s>n$ and defines a holomorphic function in this halfplane. It is known, see Ref.7 ${ }^{7}$, that the eta function extends meromorphically to the whole $s$-plane. Moreover, it is known, see Theorem 4.5 in Ref. ${ }^{7}$, that if the dimension $n$ is odd, then the eta function is holomorphic at $s=0$. This justifies, for odd $n$, the definition of the eta invariant as the real number $\eta_{H}(0)$. The eta invariant $\eta_{H}(0)$ is the traditional measure of spectral asymmetry of the operator $H$.

If we have only a finite number of eigenvalues (i.e. if we are looking at an Hermitian matrix rather than a differential operator) then the eta invariant is an integer number: it is the number of positive eigenvalues minus the number of negative eigenvalues. However, in the case of a differential operator there is no reason for the eta invariant to be integer. The basic example is that of the scalar ordinary differential operator $H(\epsilon):=-i \frac{d}{d x^{1}}+\epsilon$ acting on the unit circle parameterized by the cyclic coordinate $x^{1}$ of period $2 \pi$, with $\epsilon$ being a real parameter. It is known, see Ref. ${ }^{4}$, that the eta invariant $\eta_{H(\epsilon)}(0)$ of this ordinary differential 
operator is the odd 1-periodic function defined by the formula $\eta_{H(\epsilon)}(0)=1-2 \epsilon$ for $\epsilon \in(0,1)$. In particular, we have $\eta_{H(0)}(0)=0$ and $\lim _{\epsilon \rightarrow 0^{ \pm}} \eta_{H(\epsilon)}(0)= \pm 1$.

The current state of affairs (from an analyst's perspective) in the subject area of zeta/eta functions of elliptic operators is described in detail in the two papers Refs.16/17. Let us highlight a few facts.

- The key results are Theorem 2.7 from Ref. ${ }^{[16}$ and Proposition 2.9 from Ref. 17 . Arguing along the lines of Ref. ${ }^{7}$ one can recover from these results, in a rigorous analytic fashion, properties of the eta function.

- The eta function is holomorphic at $s=0$ in any dimension $n \in \mathbb{N}$ (i.e. without the assumption of $n$ being odd). This fact was proved by P. B. Gilkey 13 .

- The seminal paper of R. T. Seeley ${ }^{23}$ contained a small mistake: see page 482 in Ref. $\frac{16}{\square}$ or Remark 2.6 on page 39 in Ref. ${ }^{17}$ for details.

The more recent survey papers Refs. ${ }^{[4] 15}$ provide an overview of the subject.

Let us denote our massless Dirac operator on half-densities by $A(\epsilon)$, where $\epsilon \in \mathbb{R}$ is the small parameter appearing in our metric $g_{\alpha \beta}(x ; \epsilon)$. Theorem II.1 implies the following corollary.

Corollary X.1. Suppose that the coefficient c defined by formula II.5) is nonzero. Then

$$
\lim _{\epsilon \rightarrow 0} \eta_{A(\epsilon)}(0)=2 \operatorname{sign} c .
$$

Note that we have $\eta_{A(0)}(0)=0$, so formula $(\mathrm{X} .2)$ implies that the function $\eta_{A(\epsilon)}(0)$ is discontinuous at $\epsilon=0$.

Proof of Corollary X.1 Put $f(\epsilon, t):=\operatorname{Tr}\left[A(\epsilon) e^{-t(A(\epsilon))^{2}}\right]$, where $t>0$ and $\operatorname{Tr}$ is the operator (as opposed to pointwise) trace. The paper Ref ${ }^{[9}$ to which we are about to refer to actually deals with pointwise estimates, i.e. the trace in Ref. ${ }^{[9}$ is understood as the matrix trace of the integral kernel on the diagonal at a given point $x$ of the manifold. We do not need pointwise estimates for the proof of Corollary X.1.

Having fixed $\epsilon$, let us examine the behaviour of $f(\epsilon, t)$ as $t \rightarrow 0^{+}$. For a generic first order pseudodifferential operator $A(\epsilon)$ we would have $f(\epsilon, t)=O\left(t^{-2}\right)$. However, as explained in Chapter II of Ref. ${ }^{9}$, the Dirac operator in odd dimensions is very special and there are a lot 
of cancellations when one computes the asymptotic expansion for $f(\epsilon, t)$ as $t \rightarrow 0^{+}$. Namely, it was shown in Ref. 9 that

- $f(\epsilon, t)=O(\sqrt{t})$ as $t \rightarrow 0^{+}$,

- $\eta_{A(\epsilon)}(s)$ is holomorphic in the half-plane Re $s>-2$, and

$$
\eta_{A(\epsilon)}(s)=\frac{1}{\Gamma\left(\frac{s+1}{2}\right)} \int_{0}^{+\infty} t^{(s-1) / 2} f(\epsilon, t) d t \quad \text { for } \quad \operatorname{Re} s>-2 .
$$

See also Section 1 in Ref. 24 .

Formula $\mathrm{X} .3 \mathrm{p}$ implies

$$
\eta_{A(\epsilon)}(0)=\frac{1}{\sqrt{\pi}} \int_{0}^{+\infty} \frac{f(\epsilon, t)}{\sqrt{t}} d t
$$

so in order to prove Corollary X.1 we need to examine the behaviour of the integral X.4 as $\epsilon \rightarrow 0$.

Let us denote by $\lambda_{0}(\epsilon)$ the eigenvalue of the operator $A(\epsilon)$ with smallest modulus and by $E_{0}(\epsilon)$ the orthogonal projection onto the corresponding 2-dimensional eigenspace. Put

$$
\begin{aligned}
A_{0}(\epsilon) & :=\lambda_{0}(\epsilon) E_{0}(\epsilon), \quad \tilde{A}(\epsilon):=A(\epsilon)-A_{0}(\epsilon), \\
f_{0}(\epsilon, t) & :=\operatorname{Tr}\left[A_{0}(\epsilon) e^{-t\left(A_{0}(\epsilon)\right)^{2}}\right]=2 \lambda_{0}(\epsilon) e^{-t\left(\lambda_{0}(\epsilon)\right)^{2}}, \\
\tilde{f}(\epsilon, t) & :=\operatorname{Tr}\left[\tilde{A}(\epsilon) e^{-t(\tilde{A}(\epsilon))^{2}}\right]=f(\epsilon, t)-f_{0}(\epsilon, t) .
\end{aligned}
$$

Then formula (X.4) can be rewritten as

$$
\begin{aligned}
\eta_{A(\epsilon)}(0)=\frac{1}{\sqrt{\pi}} \int_{0}^{1} \frac{f(\epsilon, t)}{\sqrt{t}} d t+\frac{1}{\sqrt{\pi}} \int_{1}^{+\infty} \frac{\tilde{f}(\epsilon, t)}{\sqrt{t}} d t & \\
& +\frac{2}{\sqrt{\pi}} \int_{1}^{+\infty} \frac{\lambda_{0}(\epsilon) e^{-t\left(\lambda_{0}(\epsilon)\right)^{2}}}{\sqrt{t}} d t .
\end{aligned}
$$

The three terms in the RHS of $(\mathrm{X} .5)$ are functions of the parameter $\epsilon$ and we shall now examine how they depend on $\epsilon$.

The first term in the RHS of $(\mathrm{X} .5)$ is continuous at $\epsilon=0$ because asymptotic formulae for $f(\epsilon, t)$ as $t \rightarrow 0^{+}$are uniform in $\epsilon$. This follows from the construction of heat kernel type asymptotics for $t \rightarrow 0^{+}$: the algorithm is straightforward and examination of this algorithm shows that the asymptotic coefficients and remainder term depend on additional parameters in a continuous fashion. 
The second term in the RHS of $(\mathrm{X} .5)$ is continuous at $\epsilon=0$ because the eigenvalues of the operator $\tilde{A}(\epsilon)$ depend on $\epsilon$ continuously and because all these eigenvalues, bar one double eigenvalue, are uniformly separated from zero. The double eigenvalue in question is identically zero as a function of $\epsilon$ and does not contribute to the second term in the RHS of X.5.

Thus, the proof of formula $(\mathrm{X} .2)$ reduces to the proof of the statement

$$
\frac{2}{\sqrt{\pi}} \lim _{\epsilon \rightarrow 0} \int_{1}^{+\infty} \frac{\lambda_{0}(\epsilon) e^{-t\left(\lambda_{0}(\epsilon)\right)^{2}}}{\sqrt{t}} d t=2 \operatorname{sign} c .
$$

But formula (X.6) is an immediate consequence of formula (II.4).

The geometric meaning of the eta invariant of the Dirac operator acting over a compact oriented Riemannian manifold of dimension $4 k-1, k \in \mathbb{N}$, has been extensively studied in Refs. ${ }^{4}$. We are, however, unaware of publications dealing specifically with the Dirac operator on a 3-torus, though certain 2-torus bundles over a circle were examined in Ref ${ }^{[3}$.

\section{ACKNOWLEDGMENTS}

The authors are grateful to M. F. Atiyah, P. B. Gilkey, G. Grubb and J. D. Lotay for advice on the eta invariant, and to B. Ammann and M. Hortacsu for drawing our attention to Refs. ${ }^{12119}$.

\section{REFERENCES}

${ }^{1}$ B. Ammann, M. Dahl and E. Humbert, Surgery and harmonic spinors, Advances in Mathematics 220 (2009), 523-539.

${ }^{2}$ B. Ammann, M. Dahl and E. Humbert, Harmonic spinors and local deformations of the metric, Math. Res. Lett. 18 (2011), 927-936.

${ }^{3}$ M. F. Atiyah, H. Donnelly and I. M. Singer, Eta invariants, signature defects of cusps, and values of L-functions. Annals of Mathematics 118 (1983), 131-177.

${ }^{4}$ M. F. Atiyah, V. K. Patodi and I. M. Singer, Spectral asymmetry and Riemannian geometry. Bull. London Math. Soc. 5 (1973), 229-234.

${ }^{5}$ M. F. Atiyah, V. K. Patodi and I. M. Singer, Spectral asymmetry and Riemannian geometry I. Math. Proc. Camb. Phil. Soc. 77 (1975), 43-69. 
${ }^{6}$ M. F. Atiyah, V. K. Patodi and I. M. Singer, Spectral asymmetry and Riemannian geometry II. Math. Proc. Camb. Phil. Soc. 78 (1975), 405-432.

${ }^{7}$ M. F. Atiyah, V. K. Patodi and I. M. Singer, Spectral asymmetry and Riemannian geometry III. Math. Proc. Camb. Phil. Soc. 79 (1976), 71-99.

${ }^{8}$ C. Bär, On harmonic spinors. Acta Physica Polonica B 29 (1998), 859-869.

${ }^{9}$ J.-M. Bismut and D. S. Freed: The analysis of elliptic families. II. Dirac operators, eta invariants, and the holonomy theorem. Commun. Math. Phys. 107 (1986) 103-163.

${ }^{10}$ O. Chervova, R. J. Downes and D. Vassiliev, The spectral function of a first order elliptic system. Journal of Spectral Theory 3 (2013), 317-360.

${ }^{11}$ O. Chervova, R. J. Downes and D. Vassiliev, Spectral theoretic characterization of the massless Dirac operator. To appear in Journal of the London Mathematical Society. Available as preprint http://arxiv.org/abs/1209.3510.

${ }^{12}$ L. Erdös and J. P. Solovej, The kernel of Dirac operators on $\mathbb{S}^{3}$ and $\mathbb{R}^{3}$. Reviews in Mathematical Physics 13 (2001), 1247-1280.

${ }^{13} \mathrm{P}$. B. Gilkey, The residue of the global $\eta$ function at the origin. Advances in Mathematics 40 (1981), 290-307.

${ }^{14} \mathrm{G}$. Grubb, Analysis of invariants associated with spectral boundary problems for elliptic operators. In AMS Contemp. Math. Proc. 366 "Spectral Geometry and Manifolds with Boundary and Decomposition of Manifolds", Amer. Math. Soc., Providence (RI), 2005, $43-64$.

${ }^{15}$ G. Grubb, Remarks on nonlocal trace expansion coefficients. In Analysis, Geometry and Topology of Elliptic Operators (eds. B. Booss-Bavnbek, S. Klimek, M. Lesch and W. Zhang), World Scientific, Singapore, 2006, 215-234.

${ }^{16}$ G. Grubb and R. T. Seeley, Weakly parametric pseudodifferential operators and AtiyahPatodi-Singer boundary problems. Invent. Math. 121 (1995), 481-529.

${ }^{17}$ G. Grubb and R. T. Seeley, Zeta and eta functions for Atiyah-Patodi-Singer operators. The Journal of Geometric Analysis 6 (1996), 31-77.

${ }^{18}$ N. Hitchin, Harmonic spinors. Advances in Mathematics 14 (1974), 1-55.

${ }^{19}$ M. Hortacsu, K. D. Rothe and B. Schroer, Zero energy eigenstates for the Dirac boundary problem. Nuclear Physics B 171 (1980), 530-542.

${ }^{20}$ F. Pfäffle, The Dirac spectrum of Bieberbach manifolds. Journal of Geometry and Physics 35 (2000), 367-385. 
${ }^{21}$ F. Rellich, Perturbation theory of eigenvalue problems. Courant Institute of Mathematical Sciences, New York University, 1954.

${ }^{22} \mathrm{Yu}$. Safarov and D. Vassiliev, The asymptotic distribution of eigenvalues of partial differential operators. Amer. Math. Soc., Providence (RI), 1997, 1998.

${ }^{23}$ R. T. Seeley, Complex powers of an elliptic operator. In Proc. Symp. Pure Math. 10, Amer. Math. Soc., Providence (RI), 1967, 288-307.

${ }^{24} \mathrm{~K}$. P. Wojciechowski, The $\zeta$-determinant and the additivity of the $\eta$-invariant on the smooth, self-adjoint Grassmannian. Commun. Math. Phys. 201 (1999) 423-444. 